\title{
„Mitten auf dem Areopag“. Überlegungen zu den Schnittstellen zwischen antiker Philosophie und Neuem Testament
}

\author{
Vollenweider, Samuel
}

\begin{abstract}
In front of the Areopagus speech of Acts 17 this essay queries which historical and hermeneutical aspects are to be considered when one deals with the relationship of ancient phi-losophy and early Christian literature. With that, different perspectives must be joined: Both, Christian reception of philosophical contents and methods on one hand and ap-proaches for an explicit dialogue respectively controversy on the other hand, can be drawn into the discourses in the overarching context of Hellenistic culture, in which both philoso-phy and Christianity present themselves as guides to an extensive orientation of life.
\end{abstract}

Posted at the Zurich Open Repository and Archive, University of Zurich ZORA URL: https://doi.org/10.5167/uzh-63988

Journal Article

Originally published at:

Vollenweider, Samuel (2012). „Mitten auf dem Areopag“. Überlegungen zu den Schnittstellen zwischen antiker Philosophie und Neuem Testament. Early Christianity, 3(3):296-320. 
Samuel Vollenweider Early Christianity 3 (2012), 296-320

\section{„Mitten auf dem Areopag“6 \\ Überlegungen zu den Schnittstellen zwischen antiker Philosophie und Neuem Testament}

In front of the Areopagus speech of Acts 17 this essay queries which historical and hermeneutical aspects are to be considered when one deals with the relationship of ancient philosophy and early Christian literature. With that, different perspectives must be joined: Both, Christian reception of philosophical contents and methods on one hand and approaches for an explicit dialogue respectively controversy on the other hand, can be drawn into the discourses in the overarching context of Hellenistic culture, in which both philosophy and Christianity present themselves as guides to an extensive orientation of life.

Die plastische Erzählung der Apostelgeschichte, in der der lukanische Paulus auf dem Areopag den massgeblichen Philosophen Athens die Wahrheit des „unbekannten Gottes“ verkündigt (17,16-34), ist zu einer Schlüsselszene für das Selbstverständnis des antiken Christentums und darüber hinaus für das kulturelle Gedächtnis Europas geworden. ${ }^{1}$ Durch Lokalkolorit, Handlungsabfolge, Gesprächsführung, Topoi und Dichterzitat gibt Lukas seiner Leserschaft zu verstehen, dass es zwischen antiker Weisheit und christlicher Botschaft Schnittstellen gibt, die dem Evangelium jene bedeutungsvolle Funktion für Wahrheitserkenntnis und Lebensorientierung sichert, die auch die Philosophie beansprucht.

1 Für die Rezeptionsgeschichte von Apg 17 fehlt m.W. eine Monographie für die Alte Kirche und überhaupt eine umfassende Darstellung. Einige Hinweise zur antiken Wirkungsgeschichte bietet M. FIEDROWICZ, Die Rezeption und Interpretation der paulinischen Areopag-Rede in der patristischen Theologie, in: TThZ 111 (2002), 85-105. É. BEURLIER, Saint Paul et L'Aréopage, in: RHLR 1 (1896), 344-366 äussert sich nur zur Tribunalfrage; gar keine wirkungsgeschichtlichen Informationen bietet M.R. FAIRCHILD, Art. Areopagus, in: EBR 2 (2009), 694-697. Mit zur Wirkungsgeschichte von Apg 17 zählen Produktion und Rezeption des Corpus Dionysiacum sowohl in der östlichen wie in der westlichen Kirche. 
Die verschiedenen Facetten zwischen Konvergenz und Differenz, zwischen „Anknüpfung“ und „Widerspruch“, die die Inszenierung auf dem Areopag charakterisieren, setzen sich in den vielfältigen Konfigurationen fort, die urchristliche Verkündigung und philosophisches Denken in ihrem je spezifischen Anspruch auf die „Wahrheit“" aufeinander beziehen und voneinander trennen - Athen und Jerusalem, Gott der Philosophen und Gott der Väter, Erkennen und Glauben. Sie erstrecken sich von den Perspektiven, die die christlichen Apologeten des zweiten Jahrhunderts eröffnen, über die grossen mittelalterlichen Debatten rund um das Verhältnis der Philosophie zur Theologie und die konfessionellen Kontroversen des 16./17. Jahrhundert bis hin zur Enthellenisierungsdiskussion der Neuzeit. Beispielhaft dafür stehen die Turbulenzen, die 2006 die Regensburger Rede von Papst Benedikt XVI. mit ihrem kontroverstheologischen Profil erzeugt hat. Für das Selbstverständnis aussereuropäischer Kirchen und ihre Suche nach angemessenen Formen christlicher Inkulturation schliesslich geht die Überwindung eurozentrischer Strukturen Hand in Hand mit der theologischen Dekonstruktion von Antike und Christentum.

Die spannungsreiche Begegnung zwischen Evangelium und Philosophie in den ersten Jahrhunderten unserer Zeitrechnung bildet zwar nur einen Teilaspekt der komplexen Wechselwirkungen zwischen hellenistisch-römischer Kultur und urchristlicher Religion, die das antike Christentum als Ganzes hervorbringen, stellt aber mit ihrer Fokussierung auf konvergierende oder konkurrierende Geltungsansprüche vor eine Vielzahl von hermeneutischen Fragen. Es ist nicht zuletzt die Theologie selber, die in ihrem Namen und ihrer Geschichte Blaise Pascals programmatische Unterscheidung zwischen dem Gott der Philosophen und dem Gott Abrahams, Isaaks und Jakobs wachhält.

In den nachstehenden Zeilen soll es lediglich um einen einzelnen Aspekt dieser umfassenden Problemkonstellation gehen. Ich beschäftige mich mit der Frage, welche Gesichtspunkte historischer, exegetischer und hermeneutischer Natur zu beachten sind, wenn wir die grosse Frage nach dem Verhältnis zwischen antiker Philosophie und frühchristlicher Theologie aufwerfen und ihre Schnittstellen identifizieren. Im ersten, einleitenden Teil zeigt sich, dass die Beschränkung auf das erste Jahrhundert die Verhältnisbestimmung zugleich vereinfacht und verkompliziert. In den folgenden Teilen wird versucht, die Bezüge durch drei einfache Muster zu beschreiben - Rezeption, Dialog, Diskurs. Am Schluss werden wir nochmals kurz auf die Areopagrede zurückkommen, die jeweils auch den Untertiteln als Mottospender dient.

\section{I. „Täglich unterhielt er sich mit den Vorübergehenden“ - Kultur- geschichtliche und methodische Überlegungen}

Die Texte, die im Neuen Testament überliefert sind, dokumentieren noch nicht die Typen von Auseinandersetzung mit der Philosophie, die ab dem zweiten Jahrhundert in 
Gestalt der apologetischen Werke neue Genres in der christlichen Literaturgeschichte initiieren. Umgekehrt sind sie aber auch nicht einfach kontaktlos zur zeitgenössischen Philosophie. Insbesondere zur Stoa und zum Platonismus führen zahlreiche Brücken. ${ }^{2}$ Ein besonders herausragendes Indiz dafür ist die patristische Rezeptionsgeschichte einzelner Passagen, die die in den urchristlichen Schriften angelegten Schnittstellen identifiziert und ausweitet. Die nähere Bestimmung solcher Schnittstellen wirft allerdings erhebliche Fragen auf. Ich nenne vier Punkte.

1. Das Profil der philosophischen Kontexte, die für neutestamentliche Texte relevant sind, muss so genau wie möglich beschrieben werden. Philosophie ist in der kaiserzeitlichen Gesellschaft ein überaus vieldimensionales Unternehmen; das Spektrum reicht von elitären Philosophenschulen über Wanderprediger und Gruppen verschiedener sozialer und religiöser Konturen - fassbar beispielsweise in jenen Strömen, die die „Platonic Underworld“ hermetischer und gnostischer Zirkel bilden $-{ }^{3}$ bis zu unscharfem weltanschaulichem Material, das weit von seinem Ursprung entfernt in ganz andere Kreise und soziale Niveaus diffundiert. Von Haus aus philosophisches Gut wie Lehrsätze und Sentenzen werden durch Schule, Bildung und Markt weit herum gestreut. Es ist schwierig, generelles weltanschauliches Standardwissen als Teil eines umfassenden kulturellen Commonsense von spezifischen Figuren und Doktrinen distinkter philosophischer Herkunft zu unterscheiden.

2. Im Bereich des frühen Christentums bewegen wir uns im Raum einer Subkultur, die in mehr oder weniger starker Wechselwirkung mit frühjüdischen Milieus steht. Die erhaltene Literatur des griechischsprachigen Judentums zeigt, dass Philosophie nicht nur in Gestalt philosophischer Topoi und Figuren, sondern teilweise sogar wie im Fall Philons durch den Import umfassender Systembildung rezipiert wird. Empfängt das Christentum philosophisches Gut durch jüdische Vermittlung, ist es bereits im Vorfeld zu entscheidenden Filterungs- und

2 Zur Forschungsgeschichte vgl. für die Stoa M.L. ColisH, Stoicism and the New Testament, in: ANRW II/26.1 (1992), 334-379.

3 Vgl. J. DiLlon, The Middle Platonists. 80 B.C. to A.D. 220, Ithaca ${ }^{2} 1996,384-396 ; 450-452$. 
Transformationsvorgängen gekommen, die den Transfer in die eigene Textproduktion und Reflexion massgeblich steuern.

3. Innerhalb des Neuen Testaments gibt die Briefliteratur die stärkste Verdichtung möglicher oder wahrscheinlicher Schnittstellen mit der antiken Philosophie zu erkennen. Dieser signifikante Sachverhalt wirft die Frage nach der Rolle auf, die philosophische Prämissen und Topoi in den nur schwer erschliessbaren Kommunikationsprozessen zwischen Briefverfassern und Empfängern gespielt haben. Die Diskussion über die paulinische Korintherkorrespondenz zeigt, dass Modelle, die Paulus lediglich auf korinthische Schlagwörter und Parolen reagieren lassen, den Stellenwert philosophischer Überzeugungen viel geringer einschätzen als solche, die Paulus selber als philosophischen Lehrer porträtieren.

4. Kulturwissenschaftliche Perspektiven auf den gesamten Mittelmeerraum des späten Hellenismus und der frühen Kaiserzeit haben in den letzten Jahrzehnten erlaubt, ältere Frontstellungen abzubauen und durch produktivere Fragestellungen zu ersetzen. $\mathrm{Zu}$ denken ist insbesondere an die Abkehr vom „Judaism/Hellenism Divide“, ${ }_{4}$ der die Debatten im 19. und v.a. im 20. Jahrhundert dominiert hat, etwa in der scharfen Alternative zwischen ,hellenistischem Synkretismus“ und „weisheitlich-apokalyptischem Judentum“. Der Ausgangspunkt von einer umfassenden antik-mediterranen Globalkultur, die sich in zahlreiche miteinander wechselwirkende ethnische und soziale Partialkulturen ausdifferenziert, erlaubt es, die Kontexte, in die die urchristlichen Texte eingebettet sind, angemessener zu beschreiben. Allerdings ist man auch bei der kulturwissenschaftlichen Rekontextualisierung unserer Texte gut beraten, die Trennschärfe, die die ältere Analyse der religionsgeschichtlichen Hintergründe ausgezeichnet hat, nicht leichtfertig $\mathrm{zu}$ verspielen.

Wir versuchen im Folgenden, anhand eines schlichten Schematismus drei Typen von Verhältnisbestimmungen zwischen urchristlicher Literatur und antiker Philosophie zu unterscheiden. ${ }^{5}$ Bei der Rezeption ist davon auszugehen, dass philosophische Themen und Funktionen durch mehr oder weniger direkten Import oder auf dem Weg kultureller Diffusion Eingang finden in unsere Texte. Beim Dialog sind im Ansatz explizite christliche Bezugnahmen auf die Philosophie im Blick; als Optionen

4 Vgl. den programmatischen Band von T. EngBerg-PederSEN (Hg.), Paul beyond the Judaism/Hellenism Divide, Louisville u.a. 2001.

5 Eine etwas anders angelegte Typologie bietet D.T. RUNIA, Ancient Philosophy and the New Testament. ,Exemplar' as Example, in: A.B. MCGOwAN / K.H. RICHARD (Hg.), Method and Meaning. FS H.W. ATTRIDGE, Atlanta 2011, 347-361. 
bieten sich Anerkennung, Überbietung und Ablehnung an. Das Stichwort Diskurs bezieht sich schliesslich auf umfassende Konzeptionen der Lebensorientierung sowohl auf Seiten der Christengemeinden wie der Philosophenschulen, die auf dem weltanschaulichen Marktplatz der antiken Städte in Konkurrenz zueinander treten, ohne dass es zu Interferenzen kommen muss. Selbstverständlich bestehen Wechselwirkungen zwischen diesen drei Aspekten. Mehr noch: Die zuletzt genannte Diskurs-Perspektive steht für übergreifende kulturelle Zusammenhänge, die allererst spezifische Rezeptionen und potentielle Dialoge ermöglichen.

\section{II. "Wie auch einige von euch sagten" - Rezeption}

Die Herkunft der von Haus aus philosophischen Topoi, die uns in den urchristlichen Texten begegnen, lässt sich nicht präzis identifizieren. ${ }^{6}$ Dennoch kann man mehr oder weniger deutlich eine traditionsgeschichtlich zu beschreibende Abhängigkeit verzeichnen, also eine Art ,Genealogie“ im Unterschied zur phänomenologisch zu erhebenden Analogie. Meist erfolgt die Rezeption auf dem Weg kultureller Diffusion. Im Folgenden werden wir uns mit Zusammenhängen beschäftigen, in denen es um Inhalte (Topik), Methode (Didaktik) oder Funktion (Auftritt) geht.

\section{Topik}

1. Am meisten Aufmerksamkeit haben seit alters die zahlreichen Topoi, Themen, Motive und Denkfiguren gefunden, die die urchristlichen Texte mit der zeitgenössischen Philosophie verbinden. Das Spektrum ist differenziert, es reicht von der Gotteslehre und Christologie über die Kosmologie und Anthropologie bis zur Ethik und Politik. ${ }^{7}$ Die Bezüge erschöpfen sich nicht in peripheren Anleihen, sondern geben streckenweise die Rezeption umfassenderer Kontexte zu erkennen. ${ }^{8}$ Dabei kommt es meist zu einem mar-

6 Die bezeichnende Ausnahme bildet das Zitat aus Aratos' Phainomena, das sich Apg 17,28 findet, wohl vermittelt durch ein Florilegium. In der vom Neuen Testament unabhängigen griechischen Literatur findet sich das entsprechende Zitat nur noch bei Aristobul, frg. 4 (= PVTG 3, 223).

7 Am Rand ist allenfalls auch die Logik mit zu berücksichtigen, vgl. M. MAYORDOMO, Argumentiert Paulus logisch? Eine Analyse vor dem Hintergrund antiker Logik (WUNT 188), Tübingen 2005, 23-26.

8 Vgl. das bekannte Votum von G. BoRnKAMM, Gesetz und Natur (Röm 2,14-16), in: Ders., Antike und Christentum. Gesammelte Aufsätze Bd. 2 (BEvTh 28), München ${ }^{2} 1963,93-$ 118, hier 111: Paulus ist der Lage, ,die Gedanken der griechischen Tradition, und zwar nicht nur eklektisch und mit blossen Anleihen an ihr Vokabular, sondern durchaus in ihrem inneren Zusammenhang und ihrem sachlichen Gefüge, aufzunehmen“. Deutlich zurückhal- 
kanten Transfer in eine grundsätzlich andere theologische Konfiguration, der mit einer Umdeutung der ursprünglichen Topik Hand in Hand geht.

Das Gesagte gilt in besonderem Mass für die Briefliteratur. Wir vergegenwärtigen uns den Sachverhalt anhand einiger klassischer Beispiele bei Paulus. ${ }^{9}$ Er platziert im Römerbrief gezielt eine Serie philosophisch einschlägiger Figuren, die er in das Rahmenwerk seiner apokalyptischen Eschatologie einzeichnet und damit neu kontextualisiert. So arbeitet er in 1,19f mit dem bekannten Muster antiker natürlicher Theologie, das den Urheber aus seinen Werken erschliesst, ${ }^{10}$ konvertiert es aber zu einem Argument für die Universalität der menschlichen Verblendung. Das von Haus aus naturrechtliche Schema des universalen Gesetzes, das im Inneren der Menschen eingeschrieben ist $(2,14 \mathrm{f}),{ }^{11}$ zielt auf die Egalisierung von Juden und Heiden vor Gottes Thron. Das Elend des unerlösten Menschen wird in 7,14-25 illustriert mit dem dramatischen Kolorit, das einer dualistisch orientierten philosophischen Deutung des Konflikts der Medea entstammt. ${ }^{12}$ Mit dem ,inneren Menschen“ (7,22; vgl. 2Kor 4,16; Eph 3,16) referiert Paulus auf ein klassisches Muster ursprünglich platonischer Provenienz, ${ }^{13}$ das nun auf jeweils verschiedene Art in den apokalyptischen Äonenumbruch transponiert wird. Die Metaphorik vom Sterben und Neuwerden bereits im diesseitigen Leben (Kap. 6-8) ist ohne Anlehnung an platonische Sprachformen, die ihrerseits mit Mysterienterminologie operieren, schwer denkbar. Wahrscheinlich gilt dies überhaupt für eine Grundfigur der paulinischen Theologie, nämlich die

tender z.B. T. PAIGE, Art. Philosophy, in: G.F. HawthoRne / R.P. MARTIN (Hg.), Dictionary of Paul and his Letters, Leicester 1993, 713-718 (,While Paul may have used philosophical vocabulary for his apologetic or didactic purposes, he was not constrained by the content or method of the philosophies in vogue“", 718).

9 Vgl. die Dokumentation von J.P. SAMPLEY (Hg.), Paul in the Greco-Roman World. A Handbook, Harrisburg 2003.

10 Dies gilt auch im Fall einer literarischen Vermittlung durch Sap; vgl. J.A. LINEBAUGH, Announcing the Human. Rethinking the Relationship Between Wisdom of Solomon 13-15 and Romans 1.18-2.11, in: NTS 57 (2011), 214-237.

11 Vgl. dazu J.W. MARTENS, Romans 2.14-16. A Stoic Reading, in: NTS 40 (1994), 55-67.

$12 \mathrm{Zu}$ (allerdings anderen) platonischen Bezügen vgl. E. WASSERMAN, The Death of the Soul in Romans 7. Sin, Death, and the Law in Light of Hellenistic Moral Psychology (WUNT II/256), Tübingen 2008.

13 Vgl. C. MARKSCHIES, Art. Innerer Mensch, in: RAC 18 (1998), 216-312; H.D. BETZ, The Concept of the ,Inner Human Being' (o ع̌ $\sigma \omega \alpha \check{v} v \theta \varrho \omega \pi 0 \varsigma$ ) in the Anthropology of Paul, in: Ders., Paulinische Theologie und Religionsgeschichte. Gesammelte Aufsätze Bd. 5, Tübingen 2009, 23-52. 
Partizipation der Menschen an umfassenden Sphären. ${ }^{14}$ Im dritten grossen Teil von Röm stellt 12,1f programmatisch die ethische Autonomie der Glaubenden heraus, ${ }^{15}$ fundiert diese aber im neuen Äon, der im Zeichen von Jesus Christus steht. 13,1-7 bietet ein Stück politischer Philosophie, das die entsprechenden Diskurse der Zeit Neros spiegelt. ${ }^{16}$ In noch stärkerem Ausmass dokumentiert die Korintherkorrespondenz die Präsenz popularphilosophischer Topoi, Themen und Motive, die wir nicht nur dem Konto der heidenchristlichen Korinther, sondern auch dem theologischen Erkenntnisinteresse des Apostels gut zu schreiben haben. In christologischer Perspektive ist die Parataxe des einen Gottes und des einen Herrn Jesus Christus $(1$ Kor 8,6) von grösstem Gewicht; sie verschränkt das monotheistische Bekenntnis mit der subtilen Metaphysik der Präpositionen, wie sie sich in der hellenistischen Philosophie entwickelt hat (vgl. Röm 11,36). ${ }^{17}$ Das Bekenntnis selber ist eingebettet in eine Debatte über Freiheit und Sklaverei (1Kor 8-10; vgl. 7,17-24), die sich offenkundig auf einen weit gespannten Diskurs der hellenistisch-römischen Kultur zurückbezieht. ${ }^{18}$ In dieser Konfiguration ist auch die Stossrichtung der Belehrung über den Umgang mit Ehe und Sexualität einzuzeichnen (1Kor 7,29-35), ${ }^{19}$ transponiert in einen apokalyptischen Zeithorizont (V. 29a). Einen markan-

Vgl. U. SCHNELLE, Transformation und Partizipation in paulinischer Theologie, in: NTS 47 (2001), 58-75; zur „morphic language“ G.H. vAN KoOTEN, Paul's Anthropology in Context. The Image of God, Assimilation to God, and tripartite Man in Ancient Judaism, ancient Philosophy and Early Christianity (WUNT 232), Tübingen 2008, besonders 69-91. Vgl. zum ganzen Kapitel P.F. ESLER, Paul and Stoicism. Romans 12 as a Test Case, in: NTS 50 (2004), 106-124; auffallend zurückhaltend H.D. BETZ, Das Problem der Grundlagen der paulinischen Ethik (Röm 12,1-2), in: Ders., Paulinische Studien. Gesammelte Aufsätze Bd. 3, Tübingen 1994, 184-205. Zu Röm 12-15 vgl. R.M. THORSTEINSSON, Stoicism as a Key to Pauline Ethics in Romans, in T. Rasimus / T. ENGBERG-PEDERSEN (Hg.), Stoicism in early Christianity, Peabody Mass. 2010, 15-38; ders., Roman Christianity and Roman Stoicism. A Comparative Study of Ancient Morality, Oxford 2010, 89-104.

16 Vgl. S. KRAUTER, Studien zu Röm 13,1-7. Paulus und der politische Diskurs der neronischen Zeit (WUNT 243), Tübingen 2009, 225f; 250; 277f.

17 Vgl. H. DöRRIE, Präpositionen und Metaphysik, in: Ders., Platonica minora (STA 8), München 1976 124-136; S. VOLLENWEIDER, Christus als Weisheit. Gedanken zu einer bedeutsamen Weichenstellung in der frühchristlichen Theologiegeschichte, in: Ders., Horizonte neutestamentlicher Christologie (WUNT 144), Tübingen 2002, 29-51, hier 47-49; D. ZELLER, Der erste Brief an die Korinther (KEK 5), Göttingen 2010, 291.

18 Zum Kontext vgl. die Dokumentation in: S. VOLLENWEIDER (Hg.), Epiktet. Was ist wahre Freiheit? (Sapere), erscheint Tübingen 2013.

19 Vgl. W. DemIng, Paul on Marriage and Celibacy. The Hellenistic Background of 1 Corinthians 7 (MSSNTS 83), Cambridge 1995. 
ten Einfluss der politischen Philosophie gibt die Einheitsrhetorik zu erkennen, die 1Kor so gut wie Phil thematisch strukturiert. Diese führt freilich weit über philosophische Kontexte hinaus. ${ }^{20}$ Beim Einheit wie Differenz schaffenden Pneuma bietet sich zunächst ein attraktiver Brückenschlag zur stoischen Kosmologie an. ${ }^{21}$ Sieht man aber genauer zu, treten die Analogien in den Schatten so erheblicher Differenzen, dass man nicht leicht an einen philosophischen Reflex denken kann: Griechen assoziieren mit $\pi v \varepsilon \tilde{v} \mu \alpha$, „bewegter Luft“, nur schwer die personale Wirksamkeit des Geistes oder seinen anthropologischen Status; ${ }^{22}$ sie müssen den Terminus beim Transfer in den christlichen Soziolekt neu kodieren.

Im Besonderen bietet der Bereich der Ethik Kontaktflächen für die Rezeption philosophischen Traditionsguts. Die Christonomie der Glaubenden wird speziell im Galaterbrief herausgearbeitet (Gal 5,13-6,10); die Popularphilosophie stellt dafür nicht nur die grundlegende Figur der ethischen Autonomie, die Freiheit und Gesetz miteinander korreliert, ${ }^{23}$ sondern auch eine Fülle konkreter ethischer Maximen und Regeln bereit. Eigens zu nennen sind neben vielen einzelnen Motiven wie der agonistischen Metaphorik die Tugend- und Lasterkataloge. Auch die Haustafeln lassen sich hier verzeichnen, obschon die antike Ökonomik nicht nur in den Zuständigkeitsbereich der Philosophie fällt.

2. Die anhand der paulinischen Korrespondenz exemplarisch skizzierten Berührungen zwischen urchristlicher Theologie und philosophischer Theoriebildung lassen sich ohne Mühe auch in der übrigen Briefliteratur aufweisen. Für die von Kol empfohlene

20 Vgl. M.M. MitcheLl, Paul and the Rhetoric of Reconciliation. An exegetical Investigation of the Language and Composition of 1 Corinthians (HUTh 28), Tübingen 1991; S. VOLLENWEIDER, Politische Theologie im Philipperbrief?, in: D. SÄNGER / U. MELL (Hg.), Paulus und Johannes, Tübingen 2006 (WUNT 198), 457-469.

21 Vgl. T. EnGBerg-Pedersen, The material Spirit. Cosmology and Ethics in Paul, in: NTS 55 (2009), 179-197; der Ansatz wird weiter entwickelt in: ders., Cosmology and Self in the Apostle Paul. The material Spirit, Oxford 2010; auch H. SCHERER, Geistreiche Argumente. Das Pneuma-Konzept des Paulus im Kontext seiner Briefe (NTA.NF 55), Münster 2011, 110-115; 258-260; M. EBNER, Die Stadt als Lebensraum der ersten Christen. Das Urchristentum in seiner Umwelt Bd. 1 (GNT 1/1), Göttingen 2012, 287. Zur Diskussion vgl. R. Hirsch-Luipold, Rez. T. Engberg-Pedersen, Cosmology and Self in the Apostle Paul (2010), in: EC 3 (2012), 122-133.

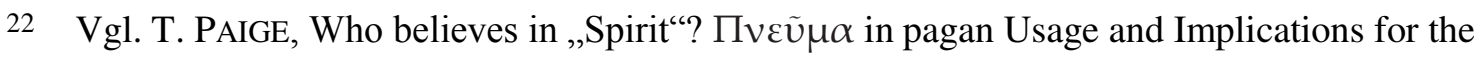
gentile Christian Mission, in: HThR 95 (2002), 417-436. Der Lateiner Seneca (besonders ep. 41,2) ist ein Sonderfall.

23 Vgl. dazu die Hinweise bei M. KonRadT, Die Christonomie der Freiheit. Zu Paulus' Entfaltung seines ethischen Ansatzes in Gal 5,13-6,10, in: EC 1 (2010), 60-81. 
Orientierung am „Oberen“ $(3,1 \mathrm{f})$ ist herkömmlicherweise die Philosophie zuständig. Das Christuslob in 1,15-20 greift ähnlich wie 1Kor 8,6 auf die philosophische Differenzierung der göttlichen Prinzipien und das damit verbundene Arrangement der Präpositionen zurück. Die am kosmischen Leib orientierte Christologie von Kol arbeitet insgesamt mit kosmologischen Figuren stoischer wie platonischer Provenienz (vgl. 2,19.17). Um die Tragweite dieser, genealogischen' Herkunftsbestimmung angemessen einschätzen zu können, muss in diesem Fall die Vermittlung durch jüdische Milieus, hier insbesondere durch die jüdische Weisheitstheologie, in Rechnung gestellt werden. Beim Eph zeigen die zahlreichen Parallelen zum Euvre Philons von Alexandria, wie hoch die vorauslaufende Rezeption philosophischen Guts durch das Judentum zu veranschlagen ist - das Spektrum reicht von der arithmetischen Theologie über die Metaphysik der Hypostasen bis zur Kosmologie.

Besonders prägnant nimmt sich die theologische Konfiguration des Hebr aus, artikuliert er doch, im Ansatz mit Philons Religionsphilosophie vergleichbar, Gottes Transzendenz und die Vermittlung zwischen Oberem und Unterem mithilfe eines mittelplatonischen Modells, ${ }^{24}$ das es auch erlaubt, überkommene religiöse Traditionen hermeneutisch zu aktualisieren. Im Blick auf die übrigen Briefe, etwa Past oder 1Petr, drängt sich allerdings erneut die Schwierigkeit in den Vordergrund, methodisch angemessen zwischen weit gestreuten kulturellen Wissensbeständen einerseits und Reflexen distinkter philosophischer Herkunft andrerseits differenzieren zu können. ${ }^{25}$ Neben der möglichen Präsenz von einzelnen Philosophumena in den Past und im 1Petr stellt sich beispielsweise die Frage, ob der Verfasser des 2Petr für seine Entfaltung der v.a. biblischjüdisch orientierten kosmologischen Eschatologie auf das spezifische stoische Lehrstück von der Ekpyrosis zurückgreift oder sich, m.E. weit wahrscheinlicher, lediglich auf rudimentäre Bestände hellenistischer Elementenphysik bezieht. ${ }^{26}$ Die „Teilhabe an der göttlichen Natur“ zusammen mit der „Flucht“ vor der kosmischen Verderblichkeit $(1,4)$ verweist ihrerseits auf platonische, nicht auf stoische Zusammenhänge.

24 W. EISELE, Ein unerschütterliches Reich. Die mittelplatonische Umformung des Parusiegedankens im Hebräerbrief (BZNW 116), Berlin 2003; K. BACKHAUS, Der Brief an die Hebräer (RNT), Regensburg 2009, 54-56; zu Hebr 8,5 vgl. RUNIA, Philosophy (s. Anm. 5).

$\mathrm{Zu} 1$ Petr vgl. die spezifischen Hinweise von R. FELDMEIER, Der erste Brief des Petrus (ThHK 15/I), Leipzig 2005, zu „Unverderblichkeit“ und „Seelenrettung“ (49-51; 58-60).

26 Die erstgenannte These wird vertreten von J.A. HARRILL, Stoic Physics, the Universal Conflagration, and the Eschatological Destruction of the „Ignorant and Unstable“ in 2 Peter, in: RASIMUS / ENGBERG-PEDERSEN, Stoicism (s. Anm. 15) 115-140. 
3. In den erzählenden neutestamentlichen Texten ist von vornherein mit weniger leicht identifizierbaren Schnittstellen zur zeitgenössischen Philosophie zu rechnen. ${ }^{27}$ Die Missionsreden des Paulus (Apg 14; 17) geben Anlass, beim lukanischen Doppelwerk als Ganzem nach philosophischen Reminiszenzen zu fragen. Lukas setzt bei seiner Leserschaft ein Bildungsniveau voraus, das in jedem Fall auch ein elementares philosophisches Training durchlaufen hat. So arbeitet seine Behandlung von Armut und Reichtum mit Elementen kynischen Gedankenguts. ${ }^{28}$

Vor allem aber steht $J o h$ im Fokus des Interesses, zumal der in seiner rezeptionsgeschichtlichen Tragweite gar nicht zu überschätzende Prolog $(1,1-18) .{ }^{29}$ So anschlussfähig sich der Logosbegriff für hermeneutische Fragestellungen und für die historischen Debatten zwischen Theologie und Philosophie seit der Alten Kirche ausnimmt, so unsicher ist es um seine philosophiegeschichtliche Genealogie bestellt. Erstens hat bereits die jüdisch-hellenistische Weisheitstheologie die Identifikation von Sophia und Logos vorgenommen. Traditionsgeschichtlich kann man sich mit guten Gründen auf dieses vermittelnde kulturelle Milieu beschränken. Zweitens täuscht die schillernde Logosprädikation leicht darüber hinweg, dass „,der Logos“ hauptsächlich in der Stoa, nicht aber in den anderen Schulrichtungen die Achse des metaphysischen bzw. kosmologischen Systems bildet. ${ }^{30}$ Drittens bleibt der Logos als hypostatisches Prinzip im Vierten Evangelium auffällig isoliert. Johanneische Kontaktflächen zu anderen stoischen Traditionen, etwa in der Pneumatologie, im Wahrheitsverständnis oder im Umgang Jesu mit den Affekten lassen sich nur mit Mühe und unter anspruchsvollen Zusatzannahmen postulieren. ${ }^{31}$ Immerhin könnte es sich lohnen, für das Verständnis der eigentümlichen johan-

$27 \mathrm{Zu}$ Jesus selber und möglichen Kynikerbeziehungen vgl. bei Anm. 39. Die Arbeiten in P.K. Moser (Hg.), Jesus and Philosophy. New Essays, Cambridge 2009, verfolgen keine traditionsgeschichtliche Fragestellung.

28 So N. NEUmanN, Armut und Reichtum im Lukasevangelium und in der kynischen Philosophie (SBS 220), Stuttgart 2010; vgl. ders., Lukas und Menippos. Hoheit und Niedrigkeit in Lk 1,1-2,40 und in der menippeischen Literatur (NTOA 68), Göttingen 2008.

29 Zur Interpretation vgl. H. WEDER, Ursprung im Unvordenklichen. Eine theologische Auslegung des Johannesprologs (BThS 70), Neukirchen 2008.

30 Zum philosophischen Stellenwert des Logos vgl. W. LÖHR, Art. Logos, in: RAC 23 (2010) 327-435, hier 328-341.

31 Pneuma: G. BuCH-HANSEN, „It is the Spirit that Gives Life“. A Stoic Understanding of Pneuma in John's Gospel (BZNW 173), Berlin 2010. - Wahrheit: P.G. KIRCHSCHLÄGER, Nur ich bin die Wahrheit. Der Absolutheitsanspruch des johanneischen Christus und das Gespräch zwischen den Religionen (HBS 63), Freiburg 2010. - Gefühle: H.W. ATTRIDGE, An „Emotional” Jesus and Stoic Tradition, in: in: RASIMUS / ENGBERG-PEDERSEN, Stoi- 
neischen Bildhermeneutik, für die „Rätselrede“ und die Symbolisierung, das Feld der philosophischen Interpretation religiöser Traditionen, von Mythen, Kulten und Bildern, zu berücksichtigen.

\section{Didaktik und Bildung}

Philosophie besteht nicht nur in Inhalten, sondern auch in Formen des Lehrens und Lernens. Die urchristlichen Texte dokumentieren zahlreiche Genera, die den Philosophen für Traditionsübermittlung und Instruktion zur Verfügung stehen. ${ }^{32}$ Was wir schon bei Topoi und Themen festgestellt haben, gilt hier in noch höherem Mass: Lehren und Lernen als entscheidendes Element der Bildung (Paideia) reicht in weite Felder des kaiserzeitlichen Stadtlebens hinein und beschränkt sich nicht allein auf die Philosophie. Die auch urchristlich belegten Sprachformen und Textsorten sind dementsprechend äusserst vielfältig; sie reichen von Traktaten über Reden bis zu Briefen; 33 an kleinen Formen ragen besonders die Sentenzen, die Chrien bzw. Apophthegmen und die Hymnen heraus. Typisch für die hellenistisch-römische Zeit sind Protreptik, Paränese und Psychagogik; man orientiert sich an Exempla und adaptiert die Inhalte konsequent und reflektiert im Blick auf die Adressatenschaft. Literarisch ragen die Diatribe ${ }^{34}$ und die Profilierung mittels Paradoxa heraus, letztere prägnant greifbar in den Peristasenkatalogen stoisch-kynischer Provenienz. In der Philosophie gewinnen zunehmend exegetische Formen an Raum; es werden nicht nur autoritative Texte, die die Schultradition konstituieren, ausgelegt, sondern auch überkommene religiöse Überlieferungen literarischer

cism (s. Anm. 15) 77-92; G. BuCH-HANSEN, The Emotional Jesus. Anti-Stoicism in the Fourth Gospel?, aaO. 93-114.

32 V.a. A.M. MALHERBE hat die ethische Belehrung und pastorale Seelsorge, die Paulus mit den Moralphilosophen seiner Zeit verbindet, herausgearbeitet: Moral Exhortation. A GrecoRoman Sourcebook, Philadelphia 1986; ders., Paul and the Popular Philosophers, Minneapolis 1989, 67-77 (,Paul's adoption, and sometimes adaptation, of the philosophical tradition, reveal to us his awareness of the philosophic pastoral methods current in his day", 68); ders., Hellenistic Moralists and the New Testament, in: ANRW II/26.1 (1992), 267-333; ders., Greco-Roman Religion and Philosophy and the New Testament, in: E.J. EPP/G.W. MACRAE (Hg.), The New Testament and its modern Interpreters (SBL.CP), Philadelphia 1989, 3-26. Eine zu scharfe Distanz zum antiken Bildungsideal baut E.A. JUDGE auf, The Reaction against classical Education in the New Testament, in: Ders., The First Christians in the Roman World (WUNT 229), Tübingen 2008, 709-716.

33 Auffällig ist das Fehlen des Dialogs, der erst ab dem zweiten Jahrhundert rezipiert wird; vgl. B.R. Voss, Der Dialog in der frühchristlichen Literatur (STA 9), München 1970. Immerhin findet sich eine Miniatur in der Areopagrede.

34 Vgl. dazu T. SchMELLER, Art. Diatribe. NT, erscheint in: EBR 5 (2012). 
oder kultisch-ritueller Art. Schliesslich ist an die Rolle der Pseudepigraphie zu erinnern, deren „Sitz im Leben“ abermals besonders die Schulinstitution bildet. Die frühchristliche Literatur kennt und benützt alle diese verschiedenen Formen; das Mass an - möglichen - philosophischen Konnotationen variiert dabei erheblich. Die Briefe stehen hier wiederum mehr im Fokus als etwa Evangelien oder Apokalypsen. So dokumentiert Matthäus zwar eine exzellente didaktische Kompetenz, greift aber kaum auf ein speziell philosophisches Lehrinstrumentarium zurück. ${ }^{35}$

\section{Auftritt}

1. Frühchristliche Gemeinden in städtischer Umgebung lassen sich in soziologischer Perspektive nicht nur mit Vereinen vergleichen. Der besondere Stellenwert, den die Christen der Lehre und dem Lernen zumessen, rückt auch die Schule in den Fokus, zumal diejenige des philosophischen Typs. Allerdings haben sich nicht alle philosophischen Richtungen schulartig organisiert - dies betrifft lediglich die „dogmatischen“ Linien, nicht aber etwa Kyniker und Skeptiker -, und die spezifische institutionelle Form ist überaus variabel, ganz abgesehen von der sozialen Stratifikation. ${ }^{36} \mathrm{Im}$ frühchristlichen Raum lassen sich schultypische Phänomene mindestens in den paulinischen Gemeinden und im johanneischen Kreis beobachten. ${ }^{37}$ In den erstgenannten zeigt sich besonders in der zweiten Hälfte des ersten Jahrhunderts und im Übergang zum zweiten Jahrhundert neben institutionellen Merkmalen (wie der deutlichen Unterscheidung von Lehrern und Schülern sowie, im Fall von Past, der Lehramtssukzession) auch der überragende Stellenwert des Schulgründers und der von ihm initiierten Tradition, fassbar insbesondere in der Pseudepigraphie (Kol, Eph; Past). Freilich muss man von einer Mehrzahl von solchen Schulen ausgehen und die jeweiligen Organisationsstrukturen

35 Anders S.K. STOWERS, Jesus the Teacher and Stoic Ethics in the Gospel of Matthew, in: RAsimus / ENGBERG-PEDERSEn, Stoicism (s. Anm. 15), 59-76, und, für die Quelle der Bergpredigt, H.D. Betz, The Sermon on the Mount. A Commentary on the Sermon on the Mount, including the Sermon on the Plain (Matthew 5:3-7:27 and Luke 6:20-49) (Hermeneia), Minneapolis 1995, 70-80 u.ö.

$36 \mathrm{Zu}$ den Philosophenschulen vgl. H. FlaSHAR / G. WÖRLER, Einleitung, in: H. FlaSHAR (Hg.), Die hellenistische Philosophie (GGPhA 4), Basel 1994, 5; A.A. LONG/D.N. SEDLEY, Die hellenistischen Philosophen. Texte und Kommentare, dt. Übs. Stuttgart 2000, 1-6; T. DORANDI, Organization and Structure of the philosophical Schools, in: K. ALGRA u.a. (Hg.), The Cambridge History of Hellenistic Philosophy, Cambridge 1999, 55-62; T. SCHMELLER, Schulen im Neuen Testament? Zur Stellung des Urchristentums in der Bildungswelt seiner Zeit (HBS 30), Freiburg 2001, 46-92.

37 Vgl. dazu besonders SchmelLeR, Schulen (s. Anm. 36); T. VegGe, Paulus und das antike Schulwesen. Schule und Bildung des Paulus (BZNW 134), Berlin 2006. 
sind noch im Fluss. Im johanneischen Bereich ist die Vergleichbarkeit mit Philosophenschulen viel geringer; ob sich die oberflächlichen Analogien zwischen den „Jesusfreunden" und dem Garten Epikurs geschichtlichen Berührungen verdanken, ${ }^{38}$ ist m.E. allein schon aus sozialgeschichtlichen Gründen zweifelhaft.

Generell markiert der faktische Status der Gemeinden als Kultvereine einen erheblichen Unterschied zu Philosophenschulen; das gilt trotz einiger bemerkenswerter Analogien (wie der kultischen Verehrung des Gründers) auch für den Garten Epikurs. Die Schwierigkeit, spezifische philosophische Einflüsse von einer weit allgemeineren kulturellen Koine unterscheiden zu können, stellt sich auch in diesem Fall; organisatorische Strukturen nach Massgabe des Schulmodells bilden sich etwa auch im medizinischen und juristischen Bereich heraus. Umgekehrt haften Elemente didaktischer oder inhaltlicher Art wie Protreptik und Psychagogik ihrerseits nicht ausschliesslich an Schulstrukturen, wie das Beispiel der Kyniker zeigt.

2. Unabhängig von dieser spezifischen institutionellen Form stehen die christlichen Missionare in der öffentlichen Wahrnehmung den umher ziehenden philosophischen Verkündigern nahe. Dies ist besonders bei Paulus der Fall; es spricht viel dafür, dass er sich mit seinem programmatischen Verzicht auf Unterhalt durch die Gemeinden - mit der Ausnahme Philippis - von den in dieser Hinsicht oft am Tropf reicher Patrone hängenden „Kollegen“ unterscheiden lassen will. Er tritt aber klar erkennbar als Lehrer auf und bildet in und neben den Gemeinden einen Mitarbeiterkreis mit deutlich schulartigen Zügen. Der Hinweis auf sein tägliches Wirken im „Lehrhaus des Tyrannus“ in Ephesus (Apg 19,9) enthält zugleich eine wertvolle historische Information wie einen typischen Zug der späteren Paulusanamnese. Der Typ des öffentlichen und, an Bedeutung ungleich gewichtiger, halböffentlichen Auftritts als Lehrer führt uns freilich einmal mehr weit über die speziell philosophischen Gemarkungen hinaus. Wenn die Evangelien, hier insbesondere Mt, Jesus als Lehrer und seine Anhänger als „Schüler“ porträtieren, legen sich nicht primär philosophische Assoziationen nahe: Mit den Bezügen zu Anhängerkreisen und Schulen von Schriftgelehrten und Rabbinen befinden wir uns wieder in distinkten jüdischen Milieus, für die die Philosophenschulen lediglich als ein formativer Faktor neben anderen in Frage kommen. An dieser Stelle ist an die besonderen Schwie 
rigkeiten zu erinnern, die galiläische Jesusbewegung trotz ihres spezifischen partialkulturellen Profils als einen Typ von kynischen Wanderphilosophen zu identifizieren. ${ }^{39}$

3. In unserem Zusammenhang verdient schliesslich ein bestimmtes Paradigma besondere Aufmerksamkeit: Sokrates als exemplarisches Bild des Philosophen schlechthin. ${ }^{40}$ Am Vorbild oder geradezu: Urbild des Sokrates haben sich nahezu alle Richtungen der antiken Philosophie orientiert. Für die christlichen Rezeptionen bietet besonders die Passion des Philosophen die Möglichkeit eines Brückenschlags zu den Märtyrern und zu Jesus selber. Die Kolorierung des Paulusbilds durch sokratische Züge begegnet nicht nur in der Areopagszene, sondern bereits bei Paulus selber, zumal in seiner Situation der Gefangenschaft (Phil 1,21-26; vgl. 2Kor 5,1-10). Auch die oben genannte Korrelation von Freiheit und Unterhaltsverzicht führt letztlich zu Sokrates zurück.

\section{III. „Einige spotteten, einige aber schlossen sich an" - Dialogische Perspektiven}

Anders als im zweiten Jahrhundert, wo die Apologeten „das Beste“ der griechischen Philosophie „für uns“ beanspruchen, kommt es in der Anfangszeit der christlichen Bewegung kaum zu einer expliziten Auseinandersetzung. Die Ausnahme bildet der Besuch des lukanischen Paulus in Athen; in gewisser Weise inszeniert die Areopagerzählung den Dialog zwischen Philosophie und Evangelium, allerdings im Modus der Überbietung (Apg 17,23b). ${ }^{41}$ Wir kommen am Ende unserer Überlegungen darauf zurück. Zwei weitere neutestamentliche Texte lassen sich unter der Fragestellung lesen, ob es hier zu so etwas wie einem verweigerten Dialog kommt, zu einer Antithese von Christusverkündigung und Philosophie.

39 Zur Diskussion vgl. H.D. BETZ, Jesus and the Cynics. Survey and Analysis of a Hypothesis, in: Ders., Antike und Christentum. Gesammelte Aufsätze Bd. 4, Tübingen 1998, 32-56; M. EBNER, Kynische Jesusinterpretation - „disciplinated exaggeration“? Eine Anfrage, in: BZ 40 (1996), 93-100.

40 Vgl. K. DöRING, Exemplum Socratis. Studien zur Sokratesnachwirkung in der kynischstoischen Popularphilosophie der frühen Kaiserzeit und im frühen Christentum (Hermes.E 42), Wiesbaden 1979; E.R. WILSON, The Death of Socrates (Profiles in History), Cambridge 2007; speziell zum Neuen Testament H.D. BetZ, Der Apostel Paulus und die sokratische Tradition (BHTh 45), Tübingen 1972; E.A. JUDGE, St Paul and Socrates, in: Ders., Christians (s. Anm. 32) 670-683.

41 Die Figur der Überbietung hat zur Folge, dass Paulus seine Dialogpartner über Erkenntnisse belehrt, die ihnen nicht nur längst bekannt sind, sondern die zu guten Teilen von ihnen selber herstammen. Mit dem „Körnerpicker“ (V. 18) wird ihm vorweg die Quittung ausgestellt! 
1. Die negative Qualifikation der „Philosophie“ in Kol 2,8 lässt sich nur unter erheblichem Vorbehalt als Polemik gegen die antike Philosophie taxieren. ${ }^{42} \phi \mathrm{t} \lambda$ oooфí $\alpha$ wird hier korreliert mit Verführung, leerem Trug, Menschenüberlieferung und den „Weltelementen“; die Antithese dazu bildet der Raum des Christus, in dem ,die verborgenen Schätze der Weisheit (бoфí $\alpha$ ) und der Erkenntnis“ beschlossen sind (2,2; vgl. 1,26-28). Der - quellensprachlich unscharfe - Begriff „Philosophie“ wird also ausgesprochen negativ konnotiert, was in der zeitgenössischen Literatur selten vorkommt. ${ }^{43}$ Viel häufiger wird ein Typ von Philosophie gegen einen anderen Typ ausgespielt, etwa in Schuldebatten oder in der Gegenüberstellung von griechischer und barbarischer Philosophie, ${ }^{44}$ oder dort, wo sie selber der Rhetorik subsumiert wird. Vor allem wird authentische Philosophie kontrastiert mit Hypokrisie; ${ }^{45}$ natürlich stellen die Philosophen selber ein dankbares satirisches Sujet dar, etwa bei Lukian und im Äsoproman. Der Verfasser von Kol schreibt diese negative „Philosophie“ seinen Gegnern zu. Angesichts der exegetischen Aporien, die bei der Rekonstruktion der kolossischen ,Irrlehre entstehen, hält man sich am besten an die elementare Regel, den Gegnern nur diejenigen Merkmale zuzuschreiben, die einerseits nicht primär polemisch kalibriert sind und andrerseits nicht paulinische Theologie fortschreiben. ${ }^{46}$ Genau letzteres ist der Fall bei den aus Gal

42 Vgl. m.R. schon Clem., strom. 1,50:5 (nur anti-epikureisch); 8,62:1 („Paulus macht offenbar der Philosophie in seinen Briefen keine Vorwürfe, sondern verlangt nur, dass, wer die Höhe des Gnostikers erreicht hat, nicht mehr auf die Stufe der griechischen Philosophie herabsinke").

43 Die bei W. BAUER, Griechisch-deutsches Wörterbuch zu den Schriften des Neuen Testaments und der frühchristlichen Literatur, hg. K. u. B. ALAND, Berlin ${ }^{6} 1988,1717$, sensu malo aufgelisteten beiden Stellen sind Fehlanzeigen: Corp. Herm. frg. 23,68 stellt Philosophie und Magie sensu bono zusammen („Philosophie und Magie nähren die Seele“); 4Makk 5,11 kontrastiert eine geschwätzige Philosophie mit einer um Wahrheit und Nutzen bemühten Philosophie.

44 Letzteres begegnet zugunsten der ,,barbarischen“ Weisheitstradition bei Tatian, or. 1-3 oder bei Iambl., myst. 7,5 (p. 258-259 des Pl.), hier allerdings ohne quellensprachliche Referenz auf „Philosophie“.

45 So z.B. Dion, or. 70,10 (,es gibt wohl kaum ein Mittel zu verhindern, dass jemand sich als

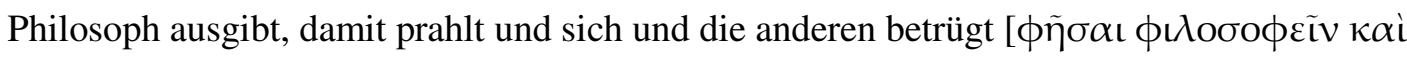

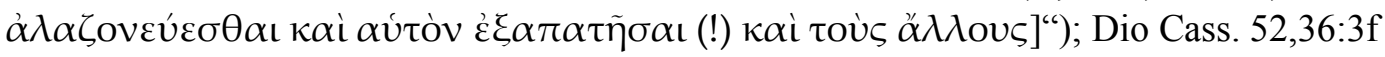
(,,vor denen, die sich als Philosophen ausgeben, hüte dich“).

46 Mit diesem Standpunkt verträgt sich die Hypothese gut, die Lokalisierung der gegnerischen Lehre in Kolossae selber als fiktionales Element zu identifizieren. Vgl. dazu N. FRANK, Der Kolosserbrief und die „Philosophia“. Pseudepigraphie als Spiegel frühchristlicher Auseinandersetzungen um die Auslegung des paulinischen Erbes, in: J. FREY u.a. (Hg.), Pseu- 
bekannten „Weltelementen“. ${ }^{47}$ Aber auch die „Philosophie“ kommt kaum als Zitat bzw. Selbstbeschreibung der kolossischen Lehre in Betracht, sondern stellt eher eine vom Verfasser von Kol ad hoc gebildete Variation zu jener Grösse dar, die Paulus selber als „Weisheit dieser Welt“" charakterisiert (1Kor 1,20f; 3,19). Die kolossische „Philosophie“ orientiert sich an den Massstäben der Menschen und der Welt statt an Christus und an Gott; sie unterscheidet sich von echter Sophia $(2,3)$ so sehr wie die ,selbstgemachte Gottesverehrung“ ( $\dot{\varepsilon} \theta \varepsilon \lambda$ anderen Worten: Der Verfasser reaktualisiert Paulus' Unterscheidung zweier Weisheiten, um der von ihm attackierten Spielart des Christusglaubens die Orientierung am „Unteren“ statt am „Oberen“ (vgl. 3,1f) zuzuschreiben.

2. 1Kor 1-4 dokumentiert einen überaus dichten Diskurs über das angemessene Verständnis von christlicher Weisheit und Philosophie. In der Korinther Gemeinde scheint es bei mindestens einer tonangebenden Gruppe ein ausgesprochenes Interesse an einer rhetorischen Weisheit gegeben zu haben, die im Schnittpunkt von griechischer und jüdischer Religionsphilosophie steht und wahrscheinlich mit der brillanten Verkündigung des Alexandriners Apollos zusammenhängt. Paulus reagiert auf diese Entwicklung, indem er seinem Aufruf an die Gemeinde, ihre Spaltungen zu überwinden $(1,10$ 4,21), eine weit ausgreifende Reflexion über zwei einander entgegengesetzte Typen von Weisheit zugrunde legt $(1,18-2,16$, umrahmt von 1,17 und 3,1-4). Er treibt die Generalisierung in 1,18-25 so weit voran, dass er „Juden“ und „Griechen“ als herausragende Repräsentanten „,dieser Welt" kontrastiert mit der Wirklichkeit des Evangeliums, in dem Gottes Macht zum Zug kommt (1,18; vgl. 1,24; 2,5). ${ }^{48}$ Die Griechen stehen im Vordergrund, da sie nach „Weisheit suchen“. Damit ist die Philosophie, ${ }^{49}$ die quellensprachlich

depigraphie und Verfasserfiktion in frühchristlichen Briefen (WUNT 246), 133-154, hier 136-142 (wonach die Gegnerkritik in 2,8 bewusst ,in einer so grundsätzlichen Weise formuliert ist, dass sie auf ein weites Spektrum möglicher Konflikt- und Bedrohungskonstellationen hin übertragbar ist", 140).

47 Mit U. LuZ, Der Brief an die Kolosser (NTD ${ }^{18} 8 / 1$ ), Göttingen 1998, $217 f$.

48 Zum Folgenden vgl. meinen Aufsatz: Weisheit am Kreuzweg. Zum theologischen Programm von 1Kor 1 und 2, in: A. DeTTWILER / J. ZuMSTEIN (Hg.), Kreuzestheologie im Neuen Testament (WUNT 151), Tübingen 2002, 43-58.

49 Vgl. z.B. Platon, rep. 5: 475b (,der Philosoph trachtet nach der Weisheit, und zwar [...]

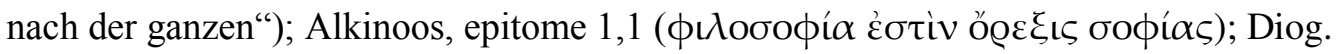

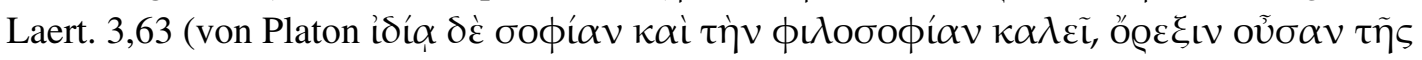

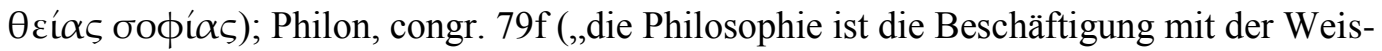
heit, die Weisheit aber die Wissenschaft von den göttlichen und menschlichen Dingen und deren Ursachen. So dürfte [...] die Philosophie auch die Sklavin der Weisheit sein"); Alex.,

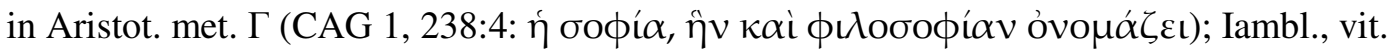


auch Theosophie u.a. umfasst und als Markenzeichen griechischer Kultur gilt, im Blick. ${ }^{50}$ Die Antithese von Weltweisheit und Kreuzeswort verkompliziert sich dadurch, dass Paulus mit zwei unterschiedlichen traditionellen Gegensatzpaaren arbeitet. Zum einen handelt es sich um die Gegenüberstellung von Rhetorik und Philosophie, die auf das klassische Griechenland zurückgeht und im Zug der zweiten Sophistik revitalisiert wird (also erst eine Generation später). ${ }^{51}$ Der Apostel setzt der Weisheitsrede aber nicht die Philosophie, sondern Kreuz $(1,17 b ; 2,1)$ und Gotteskraft $(2,4 f)$ entgegen. Zum andern greift er zurück auf eine Figur der jüdischen Apokalyptik, die die verborgene und offenbarte göttliche Weisheit mit derjenigen der Weisen kontrastiert (vgl. Dan 2,27f.30; 5,11.14f; Röm 11,33f). Dieses Gegensatzpaar überlagert dasjenige von Rhetorik und Philosophie. Der Effekt dieser Überlagerung besteht darin, dass Redekunst und Weltweisheit auf die eine Seite, Geist und göttliche Weisheit auf die andere Seite zu stehen kommen; jeder der beiden Pole hat traditionsgeschichtlich gesehen einen Bezug zur Philosophie. Deutlich ist dies zu fassen beim negativen Pol: Die Weltweisheit orientiert sich an den Ordnungen und Strukturen der gegenwärtigen Weltzeit - wozu auch Status, Prestige $(1,26)$ und menschliche Autoritätsträger (Parteienführer: 1,12; 3,5) zählen. ${ }^{52}$ Dies entspricht der Weltkonstruktion der Philosophen nach Massgabe unveränderlicher Ordnungen sowie ihrer Zugehörigkeit zur gesellschaftlichen Elite. ${ }^{53}$ Der positive Pol scheint demgegenüber kaum anschlussfähig zu sein für das Anliegen der Philosophie. Die inhaltlich als Wort vom Kreuz profilierte Weisheit Gottes bestimmt sich von der

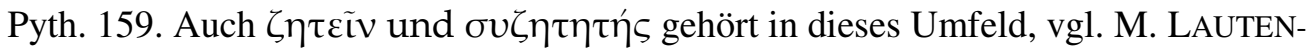

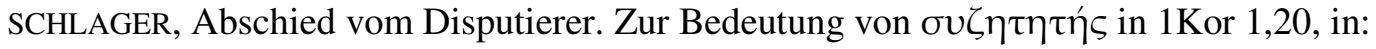
ZNW 83 (1992), 276-285.

50 Vgl. H.D. BETZ, The Gospel and the Wisdom of the Barbarians. The Corinthians' Question behind their Questions, in: Ders.: Theologie (s. Anm. 13) 88-97. (,,seeking“ wisdom constituted a common goal for the Greek intellectual culture, by the first c. A. D. shared by the Romans as well as by Hellenistic Judaism“, 94). Betz denkt, dass Paulus dem Terminus Philosophie ausweicht, weil dieser zu stark mit „scientific study of primary principles and causes" und Erwerb der Eudaimonia assoziiert werde.

51 Zum Hintergrund vgl. E. KRENTZ, Logos or Sophia. The Pauline Use of the ancient Dispute between Rhetoric and Philosophy, in: J.T. FITZGERALD u.a. (Hg.) Early Christianity and Classical Culture. FS A.J. MALHERBE (NT.S 110), Leiden 2003, 277-290.

52 Vgl. M. KONRADT, Die korinthische Weisheit und das Wort vom Kreuz. Erwägungen zur korinthischen Problemkonstellation und paulinischen Intention in 1Kor 1-4, in: ZNW 94 (2003), 181-214 (,Soziale und theologische Aspekte der Problemkonstellation sind in ihrer inneren Vernetzung zusammenzuhalten“, 213).

53 Zur „epistemological hubris“ vgl. P.W. GoOch, Paul, the Mind of Christ, and Philosophy, in: Moser, Jesus (s. Anm. 27), 84-105. 


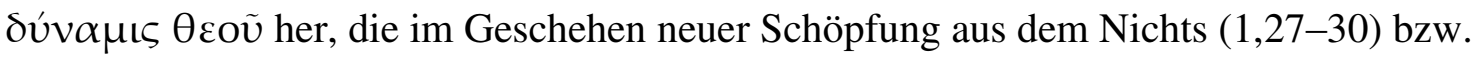
im Entstehen von Glauben (1,26a; 2,4f) wirksam ist. Sie ortet die Neues schaffende „Kraft Gottes“ im Bereich des Niedrigen, Verachteten und Schwachen, also in der Schattenzone der, Weisheit dieser Welt ${ }^{\star}$. Das Kreuz markiert den toten Winkel des philosophischen Erkenntnislichts, es steht im Zeichen der „Torheit“. Paulus bleibt aber nicht bei dieser schroffen Antithese stehen. In seiner an die „Vollkommenen“ adressierten Weisheitsrede $(2,6-16)$ reklamiert er für die Verkündigung des Evangeliums all das, was sich ein guter Teil der kaiserzeitlichen Philosophie als Weisheitsliebe nur wünschen kann: Enthüllung des Verborgenen (V. 7), übersinnliche Erkenntnis (V. 9), Erforschen der Tiefen Gottes (V. 11), epistemologisch basiert im Prinzip der Erkenntnis des Gleichen durch Gleiches (V. 11), das niveauentsprechende Erkennen (V. 13-15), schliesslich die Referenz auf den Nūs (V. 16). Allerdings legt dieser Text der philosophischen Rezeption auch enorme Hindernisse in den Weg: Die für die antike Philosophie konstitutive Bewegung des Aufstiegs scheidet vollständig aus zugunsten des katabatischen Offenbarungsvorgangs - erkennbar auch in der Ortung Gottes in der Tiefe (V. 10) -; das menschliche wie das göttliche Pneuma nimmt sich befremdlich aus. Mehr noch: Der Apostel signalisiert auch in dieser Rede an die „Vollkommenen“, dass die verborgene Weisheit Gottes in ihrem vitalen Zentrum identisch ist mit dem blossen Wort vom Kreuz (V. 12: „das, was uns Gott geschenkt hat“ [vgl. V. 9]; V. 16: Christi Geist).

Die Auseinandersetzung zwischen Paulus und den Korinthern über den Status einer christlichen Weisheit zeigt einerseits, dass sich beide Seiten im umfassenden Kontext der für die Kaiserzeit typischen philosophischen Gotteserkenntnis situieren lassen. Andrerseits formuliert Paulus ein markantes Differenzkriterium zwischen weltlicher und christlicher Weisheit: Das Kreuz polt die gesamte Konfiguration des Zusammenhangs von Gott, Welt und Menschen um. An der Christologie kommt es zum Bruch zwischen Weltweisheit und Gottesweisheit, zwischen Philosophie und Evangelium.

3. Der Dialog lebt erst im Lauf des zweiten Jahrhunderts auf, wo sich das Christentum selber als eine - das Heidentum überbietende - Philosophie zu definieren beginnt. Die Aussenwahrnehmung sieht freilich anders aus - jedenfalls dort, wo es nicht zur Konversion kommt. Es sind Philosophen, die die neue Religion (immerhin!) zum Gegenstand ihrer Aufmerksamkeit machen, um sie heftig zu attackieren. ${ }^{54}$ Dialogisch an

54 Vgl. J.G. CoOK, Some Hellenistic Responses to the Gospels and Gospel Traditions, in: ZNW 84 (1993), 233-254; ders., The Interpretation of the New Testament in Greco-Roman Paganism (STAC 3), Tübingen 2000. 
gelegte Aussenperspektiven sind ausgesprochen selten. So entdeckt im dritten Jahrhundert ein Plotinschüler, Amelios, den Johannesprolog als Dokument einer für ihn durchaus attraktiven barbarischen Weisheit. 55

\section{IV.,Was für eine neue Lehre?" - Diskurs und Komparatistik}

Unter dieser Fragestellung weiten wir die Perspektive und verbinden damit eine $m e$ thodologische Überlegung. In der älteren Forschung wurde der Sachverhalt unter dem Stichwort Analogie verhandelt, um das engere traditionsgeschichtliche, ,genealogisch“ angelegte Spender-Empfänger-Modell auf umfassendere Vergleiche hin zu öffnen. Dieses im Ansatz statische Modell lässt sich durch neuere diskursanalytische Theoriebildungen verflüssigen. Bei ,Diskursen“ handelt es sich um argumentative Dialoge über die Wahrheit von Behauptungen und die Legitimität von Normen. ${ }^{56}$ Urchristliche Stimmen beteiligen sich dieser Sicht zufolge an breiten Diskursen, die in der kaiserzeitlichen Gesellschaft des ersten Jahrhunderts geführt werden - grosse weltanschauliche Themen wie Freiheit und Schicksal, Sterben und Jenseits, Kriterien guter Lebensführung zählen ebenso dazu wie die konkreten Problemfelder von Ehe und Sexualität, von Kindererziehung, Freundschaft, Partnerverlust und Altwerden, von politischem Engagement und Berufswahl. Allerdings muss sich der trendige Diskursbegriff mit einigen Vorbehalten konfrontieren lassen. Erstens täuscht er leicht darüber hinweg, dass wir es in der Antike nicht mit derjenigen starken Präsenz von Medien zu tun haben, die für die Gutenberg-Epoche und besonders die digitalisierte Spätmoderne charakteristisch ist. Es fällt dementsprechend nicht immer leicht, für die postulierten Diskurse bis hinein in die verschiedenen Subkulturen überzeugende textliche Evidenzen beizubringen. Zweitens sind erhebliche soziale wie kulturelle Differenzen unter den Diskursgemeinschaften zu konstatieren. In unserem Fall pflegen die philosophischen Schulen Spezialdiskurse, die zwar seit späthellenistischer Zeit die Schulgrenzen deutlich überqueren - was ihnen das

55 Vgl. S. VollenweIDER, Der Logos als Brücke vom Evangelium zur Philosophie. Der Johannesprolog in der Relektüre des Neuplatonikers Amelios, in: A. DETTWILER / U. POPLUTZ (Hg.), Studien zu Matthäus und Johannes. FS J. ZUMSTEIN (AThANT 97), Zürich 2009, 377-397. Porphyrios, ebenfalls Schüler Plotins, scheint sich demgegenüber sehr negativ über Joh 1 geäussert zu haben (frg. 86 H.), vgl. CoOK, Interpretation (s. Anm. 54) $148-150$.

56 Zur Hochkonjunktur des Diskursbegriffs in den letzten Jahrzehnten vgl. die summarischen Bemerkungen von K. GRÜNDER, Vorbemerkungen, in: HWPh 9 (1995), 84-86. 
Etikett Eklektizismus verschafft -, aber relativ wenig ,Interdiskurse' mit Trägern besonderer Partialkulturen wie etwa der jüdischen ${ }^{57}$ oder dann der christlichen. Die Diskurse verlaufen vielfach nicht nur asymmetrisch, sondern bilden sogar Einbahnstrassen. Drittens, und dieses Bedenken wiegt schwer, lassen sich die Texte aufgrund ihrer jeweils sehr spezifischen impliziten Systematiken und ihrer Sondersprachen nicht leicht vergleichen; ihre Analogisierbarkeit muss erst auf dem Umweg über aufwendige Konstruktion hergestellt werden. ${ }^{58}$ Dieses Präparationsverfahren macht kulturwissenschaftliche Theoreme anfällig für den Vorwurf der Beliebigkeit. Methodisch ist die Exegese gut beraten, ihren diskursanalytischen Affirmationen durch klassische Verfahren wie Begriffsgeschichte und Motivanalyse stärkere historische Plausibilität zu verschaffen.

Im Folgenden benenne ich drei Punkte, die im Blick auf die eben verhandelte Fragestellung Aufmerksamkeit verlangen.

1. Die kaiserzeitliche Philosophie gibt in vielfacher Hinsicht Anlass, sie mit dem entstehenden Christentum zu korrelieren. Mehr als in klassischer und frühhellenistischer Zeit bietet sie eine umfassende Lebensorientierung, treibt also eine ars vivendi so gut wie eine ars moriendi. ${ }^{59}$ Philosophie und Christentum entfalten sich in einem globalen Kulturraum mit bisher nicht bekannten Graden von Individualisierung und Pluralisierung. Den Einzelnen, primär Eliten, aber zunehmend auch Mittelschichtsangehörigen, steht eine Vielzahl von Life Styles offen, für die sich Experten und praktizierende Gruppen anbieten. Dabei kommt es zu einer auffälligen Konvergenz zwischen Philosophie einerseits und jüdisch-christlicher Religion andrerseits: Auch in der Philosophie spielt

57 Die Bezugnahmen hellenistischer bzw. römischer Philosophen auf die ursprünglich jüdische griechische Bibel sind recht überschaubar; vgl. J.G. CoOK, The Interpretation of the Old Testament in Greco-Roman Paganism (STAC 23), Tübingen 2004 („,What remains unexplained is why it [sc. the LXX, S.V.] attracted so little attention from Greco-Roman intellectuals“", 345).

58 Das Paradebeispiel aus unserem Bereich stellen die innovativen Studien von T. ENGBERGPEDERSEN dar, insbesondere: Paul and the Stoics, Edinburgh 2000; zur weiteren Diskussion vgl. ders., The Relationship with Others. Similarities and Differences between Paul and Stoicism, in: ZNW 96 (2005), 35-60 (in Auseinandersetzung mit EsLER, Paul [s. Anm. 15]); ders., Cosmology (s. Anm. 21). Der Nachweis von textlich klar nachweisbaren Rezeptionen tritt in den Hintergrund zugunsten von strukturalen Entsprechungen. Im erstgenannten Buch reguliert das ,I $\rightarrow \mathrm{X} \rightarrow \mathrm{S}-$ Modell““ (d.h. Wechselwirkungen zwischen Individuum, Gott bzw. Logos und Gemeinschaft) die Präparation des stoischen und paulinischen Textmaterials (33-44).

59 Vgl. P. HADOT, Philosophie als Lebensform. Geistige Übungen in der Antike, dt. Übs. Berlin 1991. 
die Konversion eine tragende Rolle; sie geht mit einem Identitätswechsel und einem damit verbundenen Ethos einher. ${ }^{60}$ Die Entscheidung für die Philosophie bedeutet, bisherige kulturelle Standards zu verabschieden und sich auf einen neuen Way of Life einzulassen. Das Bildungsideal (Paideia), das die griechisch-römische Kultur überhaupt auszeichnet, gewinnt durch die Orientierung an der Konversion als Lebensbruch ein sehr bestimmtes Profil. Auch aus dieser Perspektive nehmen sich die Analogien im Bereich des „Auftritts“, nämlich die Selbstorganisation in Gestalt von ,Schulen“, keineswegs überraschend aus. ${ }^{61}$ Die gelegentlich von der Forschung festgestellte Nähe zwischen christlichen Gemeinschaftsformen und epikureischem Freundeskreis hat viel eher mit der Durchschlagskraft kultureller Muster als mit realen geschichtlichen Kontakten zu tun. ${ }^{62}$

2. Angesichts der benannten Analogien überrascht es nicht, dass bereits in hellenistischer Zeit Exponenten des Judentums ihre althergebrachte Religion als Philosophie präsentieren. Mose wird als Kulturbringer gefeiert, der längst vor den Griechen die Philosophie entdeckt hat. Josephus kann deshalb auch die innerjüdischen Gruppen und Strömungen als philosophische Schulrichtungen porträtieren. In der Areopagerzählung nehmen die Athener Philosophen mit Paulus als potentiellem Kollegen Kontakt auf; ab dem zweiten Jahrhundert stellt sich das Christentum bei Apologeten wie Gnostikern entsprechend dar. ${ }^{63}$ Die Interpretatio Graeca von Religion als Philosophie zeigt exemplarisch, wie sich Juden und Christen in hellenistisch-römischer Zeit auf eine weitreichende kulturelle Kontextualisierung ihrer überkommenen Traditionen eingelassen ha-

60 Vgl. die klassische Darstellung von A.D. Nock, Conversion. The Old and the New in Religion from Alexander the Great to Augustine of Hippo, Oxford 1933, 164-186; ferner C. SCHÄUBLIN, Konversionen in antiken Dialogen?, in: Ders., Aus paganer und christlicher Antike. Ausgewählte Aufsätze zur Klassischen Philologie (1970-1997), Basel 2005, 125140; I. TANASEANU-DöBLER, Konversion zur Philosophie in der Spätantike. Kaiser Julian und Synesios von Kyrene, Stuttgart 2008, 11-22; E.A. JUDGE, Conversion in the ancient World, in: Ders., Jerusalem and Athens. Cultural Transformation in late Antiquity (WUNT 265), Tübingen 2010, 261-263; J.E. SPITTLER, Art. Conversion II. Greco-Roman Antiquity, erscheint in: EBR 5 (2012).

61 Betont von S.K. Stowers, Does Pauline Christianity resemble a Hellenistic Philosophy?, in: ENGBERG-PEDERSEN, Divide (s. Anm. 4), 81-102.

62 Hierin mit C. GLAD, Paul and Philodemus. Adaptability in Epicurean and early Christian Psychagogy (NT.S 81), Leiden 1995 (,a widespread and shared communal practice among Epicureans and early Christians", 9; vgl. 335f).

63 Vgl. W. LÖHR, Christianity as Philosophy. Problems and Perspectives of an ancient intellectual Project, in: VigChr 64 (2010), 160-188. 
ben. Das Terrain dafür präpariert hat das enorme Interesse der Griechen an der „Barbarenphilosophie“".64

3. Mit dem Verständnis der Philosophie als Lebenskunst und Psychagogik rückt die Ethik in den Vordergrund, die die grundlegenden Überzeugungen für die Lebensführung relevant macht. Es überrascht nicht, dass sich im moralisch-ethischen Bereich die meisten Übereinstimmungen zwischen frühem Christentum und hellenistischer Philosophie namhaft machen lassen. Man darf aber nicht übersehen, dass gerade die Ethik hellenistischer Philosophen auf einem ontologischen Fundament aufruht, das die erhaltenen Texte nicht immer hinreichend deutlich erkennen lassen ${ }^{65}$ - auch das macht die Philosophie vergleichbar mit dem antiken Judentum so gut wie mit dem Christentum. Die Lebenskunst setzt ein Vertrauen auf den Lebensgrund voraus, der in Physik und Metaphysik zum Gegenstand der Reflexion wird. Möglich wird die ,soteriologische' Funktion der Dogmatik überhaupt erst dadurch, dass die Philosophie ab dem ersten Jahrhundert v.Chr. und verstärkt mit dem Beginn der Spätantike, d.h. ab dem späten dritten Jahrhundert, das Thema der Religion bearbeitet. ${ }^{66}$ Sie verstärkt nicht nur ihre Kompetenz für den gesamten Bereich der Theologie - von der henotheistisch profilierten Prinzipienlehre ${ }^{67}$ über die Kosmologie und Psychologie bis zur Eschatologie -, sondern bietet mit ihrer Psychagogik und Soteriologie auch Hilfestellung im Umgang mit Lebenskontingenzen (coping), die in der späteren Antike bedrohlich zunehmen. Vor allem erklärt sie das Feld der traditionellen Religionen mit ihren Texten, Mythen und Riten zu ihrem Kerngeschäft; sie profiliert sich weithin als Religionsphilosophie und setzt dafür auch die exegetischen Techniken ein, die sie für die Interpretation ihrer autoritativen

64 Zu dieser vgl. T. KoBUSCH, Christliche Philosophie. Die Entdeckung der Subjektivität, Darmstadt 2006, 51-57; A. DiHLE, Die griechische Philosophie zur Zeit ihrer Rezeption durch Juden und Christen, in: R. HIRSCH-LuIPOLD u.a. (Hg.), Religiöse Philosophie und philosophische Religion der frühen Kaiserzeit. Literaturgeschichtliche Perspektiven (STAC 51), Tübingen 2009, 3-19, hier 3-7.

65 Vgl. M. Frede, Epilogue, in: Algra, History (s. Anm. 36), 779-781.

66 Vgl. dazu D. Frede / A. LAKS (Hg.), Traditions of Theology. Studies in Hellenistic Theology, its Background and Aftermath (PhAnt 89), Leiden 2001; R. HIRSCH-LUIPOLD, Die religiös-philosophische Literatur der frühen Kaiserzeit und das Neue Testament, in: Ders., Philosophie (s. Anm. 64), 117-146; P. VAN NufFELEN (Hg.), Rethinking the Gods. Philosophical Readings of Religion in the post-Hellenistic Period, Cambridge 2011; für die hellenistische Zeit J. MANSFELD, Theology, in: ALGRA, History (s. Anm. 36), 452-478.

67 Vgl. die Dokumentationen von P. AtHAnASSiAdi / M. Frede (Hg.), Pagan Monotheism in Late Antiquity, Oxford 1999; St. Mitchell / P. VAN NufFelen (Hg.), One God. Pagan Monotheism in the Roman Empire, Cambridge 2010. 
philosophischen Texte, besonders von Platon und Aristoteles, entwickelt hat. ${ }^{68}$ In Form der neuplatonischen Theurgie stellt sie sich schliesslich sogar im Feld kultischer Performanz auf. Die Konvergenzen zwischen Philosophie und christlicher Theologie decken bereits im ersten Jahrhundert ein weites Spektrum ab.

4. So sehr sich aus der Perspektive von Diskurs und Kontext die urchristliche Religion als Einzelphänomen in einem gleichermassen globalen wie pluralen Kulturraum einzeichnen lässt, so sehr sticht die Eigenwilligkeit und Sperrigkeit der Grammatik, die ihre eigenen Diskurse bestimmt, ins Auge. Die älteren Versuche der Theologie, fundamentale Differenzen zwischen „Athen“ und „Jerusalem“ zu konstatieren, haben hier ihr bleibendes Recht. Es ist kein Zufall, dass entscheidende Schlüsselbegriffe der hellenistischen Philosophie wie Eudaimonia und Aretē im neutestamentlichen Vokabular fast ganz fehlen. Versucht man, die Gesamtkonfiguration der Spielarten frühchristlicher Theologie zu denjenigen der kaiserzeitlichen Philosophie in ein Verhältnis zu setzen, spielen insbesondere die elementaren Koordinaten von Raum und Zeit eine gewichtige Rolle. Während sich bei der symbolischen Konstruktion des Raums nennenswerte Übereinstimmungen abzeichnen - man denke nur an das Weltbild des Eph -, ${ }^{69}$ stellt die christliche Eschatologie in der Repräsentation von Zeit vor einen tiefen Graben. Auf der Linie von 1 Kor $1 / 2$ wäre dann zu fragen, ob es nicht die Christologie selber ist, ${ }^{70}$ die Verkündigung eines Gekreuzigten als endzeitlichem Erweis der $\delta \dot{v} \alpha \mu \iota \varsigma$ $\theta \varepsilon 0 \tilde{v}$, die die Grunddifferenz zwischen Philosophie und Evangelium markiert. Aber damit sind wir bereits im Feld systematischer Konstruktion angelangt, die der Vielfalt der überlieferten Texte nicht hinreichend gerecht zu werden vermag.

\section{V. "Gott zu suchen“ - Bilanz}

Wir kehren zur Areopagerzählung zurück. Sie setzt viele der von uns benannten Perspektiven in Szene. Lukas situiert sein Programm einer kontextuellen Theologie im

68 Zur „exegetischen Phase“ der hellenistischen Philosophie vgl. P. HADOT, Art. Philosophie. E. Hellenismus, in: HWPh 7 (1995), 592-599, hier 596f.

69 R. SCHWINDT, Das Weltbild des Epheserbriefes. Eine religionsgeschichtlich-exegetische Studie (WUNT 148), Tübingen 2002.

70 Vgl. dazu X. Tilliette, Le Christ de la philosophie. Prolégomènes à une christologie philosophique (CFi 155), Paris 1990. 
traditionsbewussten Athen; Paulus und die Philosophen begegnen sich auf Augenhöhe (dass der christliche Verkündiger seinen Dialogpartnern gegenüber im Gestus der Überlegenheit auftritt, ist für das antike Empfinden weniger befremdlich als für das moderne pluralistische); sein Vortrag arbeitet mit zentralen Einsichten der philosophischen Aufklärung und Theologie; er selber wird als neuer Sokrates stilisiert. ${ }^{71}$ Die Rede auf dem Areopag erlaubt auf weite Strecken hin ein double Reading - sie lässt sich sowohl aus biblisch-jüdischer wie aus hellenistisch-philosophischer Perspektive lesen und bietet damit ein Paradigma von ,Interdiskursivität': Tempel- und Kultkritik; Erkenntnis Gottes aus der Welt; Belebung des Kosmos mit Leben und Geist; Ursprung der Menschheit; Ordnung der Räume und Zeiten; Nähe Gottes und Gotteskindschaft - alle diese Theoreme lassen sich sinnvoll in beiden Konfigurationen einzeichnen, mehr noch: sie konstituieren ein übergreifendes Koordinatensystem. Prägnant wird die Konvergenz durch das Dichterzitat von V. 28 herausgestellt, an der Stelle, wo in den anderen Reden der Apg die Schrift zu Wort kommt. Zugleich markiert unser Text sehr genau den Ort, wo der Dialog abbricht: Gericht und Totenauferstehung (V. 31f; vgl. V. 18), also die Eschatologie. Dazu kommt die sokratische Szene als Ganze, die den Tod des Verkündigers einer neuen, unbekannten Gottheit in den Horizont rückt: Der Athener Diskurs findet nicht in einem herrschaftsfreien Raum statt, sondern dort, wo der Gerichtshof tagt. ${ }^{72} \mathrm{Er}$ steht somit in einem grossräumigen religionspolitischen Spannungsfeld, das Lukas in seinem Historienwerk von Anfang bis Ende explizit artikuliert (vgl. Lk 1,51f; Apg 28,31).

Der letztgenannte politische Akzent, den eine Überlegung zu den Schnittstellen zwischen Neuem Testament und antiker Philosophie nicht unberücksichtigt lassen darf, gibt uns Anlass zu einem abschliessenden Denkanstoss. Seit einiger Zeit ist ein reges Interesse von Seiten der Philosophie am Apostel Paulus zu beobachten, namentlich bei Jacob Taubes, Alain Badiou, Giorgio Agamben und Slavoj Žižek. ${ }^{73}$ Paulus interessiert

71 Zur hermeneutischen Frage nach dem Verhältnis von historischem und erinnertem Paulus vgl. J. SCHRÖTER, Konstruktion von Geschichte und die Anfänge des Christentums. Reflexionen zur christlichen Geschichtsdeutung aus neutestamentlicher Perspektive, in: Ders. / A. EDDELBÜTTEL (Hg.), Konstruktion von Wirklichkeit (TBT 127), Berlin 2004, 201-219, hier: 213-217.

72 Vgl. C.K. Rowe, The Grammar of Life. The Areopagus Speech and Pagan Tradition, in: NTS 57 (2011), 31-50, hier: 37f; 49f.

73 Vgl. zu diesem Trend B. Bourgine, Saint Paul et la philosophie. Crise du multiculturalisme et universel chrétien, in: RTL 40 (2009), 78-94; L. BORMANN, Die Radikalität des Paulus. Die neuen philosophischen Paulusinterpretationen und die neutestamentliche Wissenschaft, in: M. BRASSER (Hg.), Paulus und die Politik, Freiburg 2009, 134-155; H. SEU- 
als ein Denker, der eine Vernunft jenseits der griechischen Ontologie entdeckt, mehr noch: der eine Antiphilosophie betreibt, die im Zeichen einer ontologischen Subversion steht. ${ }^{74}$ Die Textauslegung dieser kritischen Sympathisanten Pauli ist wild und nimmt keine Rücksicht auf zünftige exegetische Standards. Sie erhebt aber einen provokativen Anspruch auf die Wahrheit der Texte des Neuen Testaments - eine scheinbar antiquierte Fragestellung, die angesichts des imponierenden Aufgebots kulturwissenschaftlicher Hermeneutik durch heutige Exegeten ins Abseits geraten ist. Unsere Beschäftigung mit den Beziehungen zwischen antiker Philosophie und urchristlicher Verkündigung könnte sich einen reichen Ertrag von der folgenden Einsicht versprechen: Die Philosophie, auch und gerade in ihrer antiken Variante, hält die Erinnerung daran wach, dass das Neue Testament zu denken gibt, dass es nicht nur auf existentielle Nachfolge zielt, sondern auch Nachdenken entfesseln will. Das hebt, neben manch anderem, das Urchristentum von den zahlreichen devianten endzeitlichen Gruppierungen ab, mit denen es soziologisch häufig verglichen wird. Das jüngste Interesse am Neuen Testament, das manche Philosophen in ihrer Frontstellung gegen die Beliebigkeit des spätmodernen Relativismus eint, steht ganz im Zeichen jener von Haus aus urphilosophischen Frage, die der Statthalter Roms, der Repräsentant ,dieser Welt', an den johanneischen Christus gerichtet hat: „Was ist Wahrheit?“ (Joh 18,38)

BERT, Politische Theologie bei Paulus? Ein neuerer philosophischer Diskurs, in: VF 55 (2010), 60-70.

74 So A. BADIOU, Paulus. Die Begründung des Universalismus, dt. Übs. München 2002, 90. „Was ihn in Athen in Schwierigkeiten bringt, ist kurz gesagt seine Antiphilosophie“, 53. 